\title{
Supermicrosurgical Lymphaticovenous Anastomosis for Subacute Lymphorrhea after Inguinal Lymph Node Biopsy: A Case Report
}

\author{
Young Chul Suh, Ji Sung Lee, Seong Yeon Kim, Young Jin Kim, Jung Ho Lee \\ Department of Plastic and Reconstructive Surgery, Bucheon St. Mary's Hospital, College of Medicine, The Catholic University of Korea, Bucheon, Korea
}

\begin{abstract}
Lymphorrhea usually arises from surgical intervention to the lymphatic system. Most lymphorrhea cases can be treated with conservative treatments, but some require further surgical intervention. The most commonly performed surgical procedure is macroscopic ablation of the subcutaneous fat tissue that seems to contain ruptured lymphatic vessels. However, ablation is not always successful because it is difficult to identify the ruptured lymphatic vessels. Furthermore, the disruption of lymph drainage results in lymphedema. Lymphaticovenous anastomosis (LVA) has multiple advantages over the other methods currently used to treat lymphorrhea. First, LVA is minimally invasive technique because it can be performed under local anesthesia and requires same small skin incisions that were made previous operation. Second, LVA is effective for all sizes of surgical fields, ranging from simple lymph node biopsy to pelvic lymphadenectomy. Most studies, however, discuss LVA as a procedure after formation of lymphocele rather than for subacute lymphorrhea. We introduce LVA as a treatment option for the subacute stage of lymphorrhea that does not respond to conservative care.
\end{abstract}

Keywords: Microsurgery, Lymph node excision, Postoperative complication, Wound healing

\section{Introduction}

Lymphorrhea can occur from any kind of surgical intervention in the lymphatic system. Conservative treatments, such as compression and aspiration of the retained lymph fluid, are usually effective. Some cases, however, are refractory to conservative treatments and require further surgical interventions [1]. Sclerotherapy has been proposed, and several sclerosant agents, such as bleomycin, OK-432, polidocanol, sodium tetradecyl sulfate, trisodium citrate, fibrin glue, ethanol, and tetracycline, have been used, mainly for pelvic lymphorrhea. This therapy has the advantage of being very simple but the drawback is that these agents cause inflammation of the lymphocele walls and the lymphatic vessels, leading to fibrosis [2]. This type of local fibrosis can induce permanent obliteration of the distal lymphatic system, resulting in lymphedema [3]. Lymphaticovenous anastomosis (LVA) using supermicrosurgery has been reported as a simple, minimally invasive, and effective treatment for secondary lymphedema of the upper and lower extremities [4-6]. This technique bypasses the proximal lymphatic blockages, providing an alternate route for lymphatic fluid recirculation into the venous system. We used this supermicrosurgical technique for inguinal lymphorrhea. By providing an alternate route of lymphatic drainage for the lymphatic fluid from the lower leg into the venous system, lymphorrhea would be reduced.

\section{(1) \\ Case Report \\ Received: July 11, 2018 \\ Revised: August 28, 2018 \\ Accepted: August 28, 2018 \\ Corresponding author: \\ Young Chul Suh, M.D. \\ Department of Plastic and Reconstructive Surgery, Bucheon St. Mary's Hospital, College of Medicine, The Catholic University of Korea, 327 Sosa-ro 327beon-gil, Wonmi-gu, Bucheon 14647, Korea \\ E-mail: ycsuh@catholic.ac.kr}

This is an Open Access article distributed under the terms of the Creative Commons Attribution Non-Commercial License (http://creativecommons.org/licenses/by-nc/4.0/) which permits unrestricted non-commercial use, distribution, and reproduction in any medium, provided the original work is properly cited.

(c) 2018 Korean Wound Management Society 


\section{Case}

A 53-year-old woman was referred to the Department of Plastic Surgery for a lymph node biopsy for the diagnosis of multiple myeloma. Based on an enhanced pelvic computational tomographic (CT) scan and an indigo carmine dye injection into the anterior superior iliac spine, we localized a superficial lymph node, only half of which was resected for biopsy to prevent post-biopsy lymphedema (Fig. 1). The biopsy result came back as negative for malignancy, but the patient was diagnosed with reactive hyperplasia. However, the wound did not heal because of continued lymphorrhea despite conservative treatments, including compression and manual drainage for 12 days.

Fourteen days after the lymph node biopsy, after performing

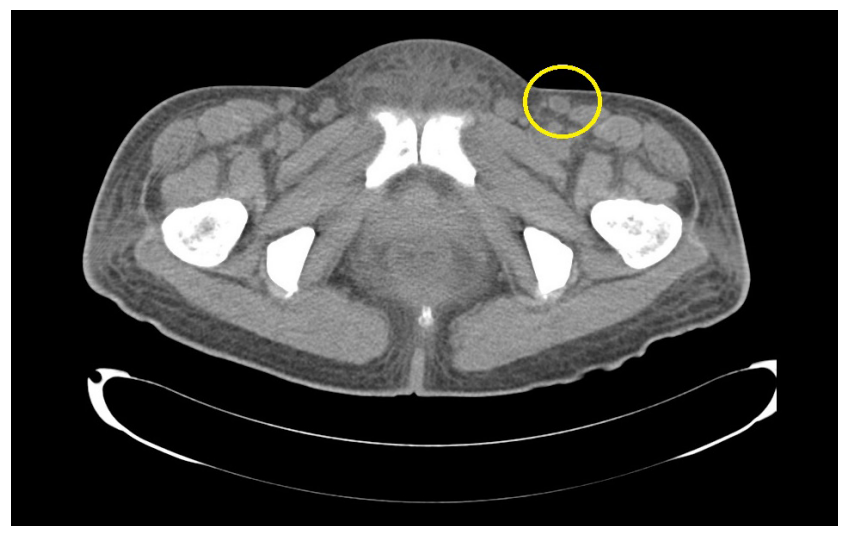

Fig. 1. Computed tomography scan performed before lymph node biopsy. We could localize superficial lymph node preoperatively.

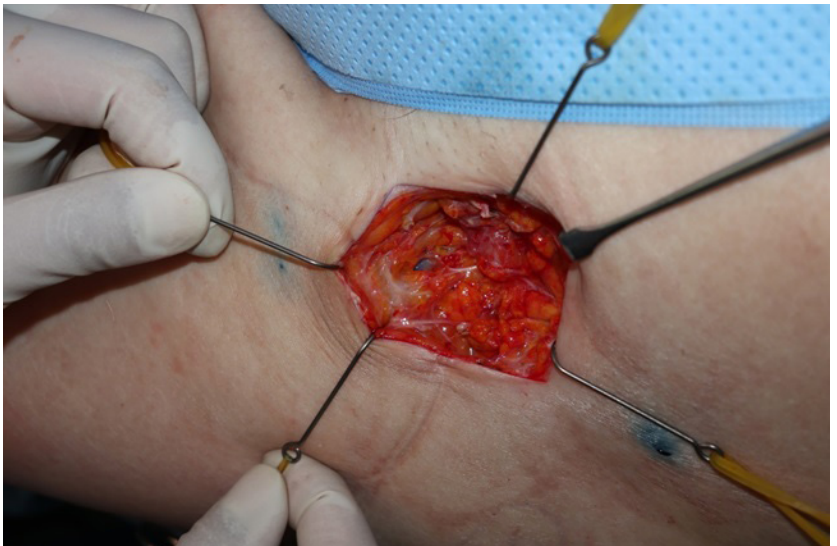

Fig. 2. Indigo carmine dye injection. Confirmation of the exact source of lymphorrhea by subcutaneous indigo carmine dye injection. intraoperative indocyanine green (ICG) lymphography, along with a blue dye injection, LVA was performed under local anesthesia (Fig. 2). Under intraoperative ICG lymphography, a ruptured lymphatic vessel and a nearby healed lymph node were observed and dissected for anastomosis. An end-to-end LVA with intravascular stent technique using 5-0 nylon suture was performed between the lymphatic vessel and a superficial vein in the left inguinal area [7] (Figs. 3, 4). The caliber of the lymphatic channel was $0.6 \mathrm{~mm}$. The postoperative ICG lymphography revealed intact lymph flow from the lymphatic vessel to the superficial vein (Fig. 5). The postoperative course was uneventful during two months' follow-up, and lymphorrhea had completely disappeared from the first day after the procedure.

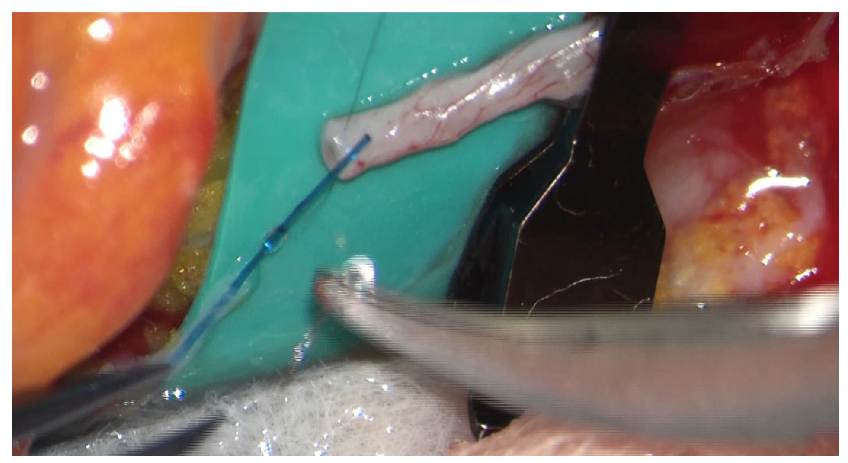

Fig. 3. IVAS technique. LVA using intravascular stent technique with 5-0 Nylon.

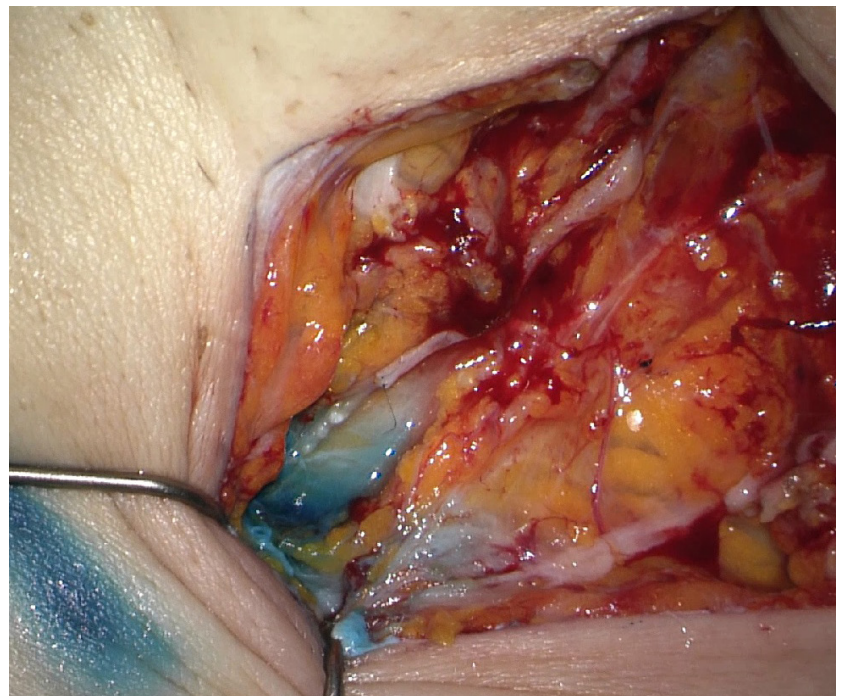

Fig. 4. Photographs obtained after LVA. The caliber of the lymphatic channel was $0.6 \mathrm{~mm}$. 


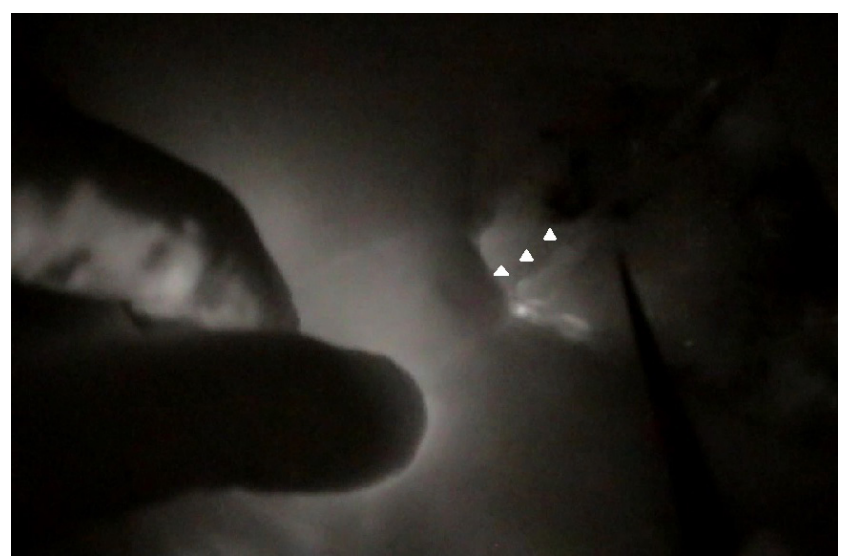

Fig. 5. Post LVA ICG lymphography. White triangles indicate lymphatic flow along the microanastomosis site.

\section{Discussion}

Iatrogenic lymphorrhea occurs after surgical intervention in the lymphatic system. Most lymphorrhea cases are responsive to conservative treatments such as compressive dressing, but some require further surgical treatments $[8,9]$. The most commonly performed surgical procedure is the macroscopic ablation of subcutaneous fat tissue that seems to contain the causative ruptured lymphatic vessels. However, macroscopic ablation is not always effective because it is difficult to identify the ruptured lymphatic vessels exactly. Furthermore, the disruption of lymph drainage can result in lymphedema [10]. Continued lymphorrhea can lead to a non-healing wound in surgical sites, or the formation of a lymphocele. The ideal therapy for lymphorrhea should be more effective and less invasive than traditional treatment methods (including sclerotherapy and fibrin glue), with fewer complications and a lower chance of recurrence. Moreover, the restoration of the lymphatic circulation, disrupted by lymph node biopsy or lymphadenectomy, is desired. LVA is emerging as the treatment of choice for lymphedema of the extremities. Before the LVA operation was available, only conservative therapeutic modalities, such as massage and compression, could be used for lymphedema [4]. These techniques do not enable re-establishment of lymphatic circulation into the venous system, rather they simply release the lymph into the trunk region. In contrast, LVA is able to restore the physiological association between the lymphatic and the venous systems, which allows us to use this technique on patients with lymphorrhea unresponsive to conservative therapy.

In our case, we performed LVA for lymphorrhea in the sub- acute phase that has seldom been discussed previously. We understood from the quick lymphatic leakage after the blue dye injection and the ICG lymphography findings that the lymphatic vessel involved in the development of lymphorrhea had a strong lymphatic flow. Therefore, we decided not to follow the sclerotherapy strategy since in our opinion, the definitive interruption of such a lymphatic vessel, could have caused an impairment in the lymphatic flow, with the potential possibility of lymphedema or lymphocele occurrence.

LVA has multiple advantages over the other methods currently used to treat lymphorrhea. First, LVA is minimally invasive technique because it can be performed under local anesthesia through incisions that were made during previous operations. Second, LVA is effective for all types of surgical fields, ranging from simple lymph node biopsy to pelvic lymphadenectomy. Third, LVA can prevent or improve lymphocele which is a common complication of lymphatic disruption. This is in contrast to other techniques that resolve the fluid collection by blocking or sclerosing lymphatic channels, which may in itself provoke the development of lymphocele [6].

Currently, there is no clinical guideline for the treatment of lymphorrhea after lymph node biopsy or lymphadenectomy. Though there are only limited conservative options for the treatment of lymphorrhea, most studies discuss LVA as a procedure after formation of lymphocele rather than for subacute lymphorrhea $[1,3,9,10]$. This indicates that surgeons may wait for several months before deciding to perform LVA. However, LVA is currently available as a potent option for the treatment of subacute lymphorrhea if sclerotherapy has not been used. A further clinical trial is warranted to compare the efficacy of several different options for treating subacute lymphorrhea.

\section{Conflict of interest}

No potential conflict of interest relevant to this article was reported.

\section{Ethical approval}

The study was performed in accordance with the principles of the Declaration of Helsinki. Written informed consents were obtained.

\section{Patient consent}

The patients provided written informed consent for the publication and the use of their images. 


\section{References}

1. Yamamoto T, Yoshimatsu H, Koshima I. Navigation lymphatic supermicrosurgery for iatrogenic lymphorrhea: supermicrosurgical lymphaticolymphatic anastomosis and lymphaticovenular anastomosis under indocyanine green lymphography navigation. J Plast Reconstr Aesthet Surg 2014;67: 1573-9.

2. Gentileschi S, Servillo M, Salgarello M. Supramicrosurgical lymphatic-venous anastomosis for postsurgical subcutaneous lymphocele treatment. Microsurgery 2015;35:565-8.

3. Roberts JR, Walters GK, Zenilman ME, et al. Groin lymphorrhea complicating revascularization involving the femoral vessels. Am J Surg 1993;165:341-4.

4. Koshima I, Inagawa K, Urushibara K, et al. Supermicrosurgical lymphaticovenular anastomosis for the treatment of lymphedema in the upper extremities. J Reconstr Microsurg 2000;16:437-42.

5. Koshima I, Nanba Y, Tsutsui T, et al. Minimal invasive lymphaticovenular anastomosis under local anesthesia for leg lymphedema: is it effective for stage III and IV? Ann Plast
Surg 2004;53:261-6.

6. Todokoro T, Furniss D, Oda K, et al. Effective treatment of pelvic lymphocele by lymphaticovenular anastomosis. Gynecol Oncol 2013;128:209-14.

7. Narushima M, Mihara M, Yamamoto Y, et al. The intravascular stenting method for treatment of extremity lymphedema with multiconfiguration lymphaticovenous anastomoses. Plast Reconstr Surg 2010;125:935-43.

8. Twine CP, Lane IF, Williams IM. Management of Iymphatic fistulas after arterial reconstruction in the groin. Ann Vasc Surg 2013;27:1207-15.

9. Van den Brande P, von Kemp K, Aerden D, et al. Treatment of lymphocutaneous fistulas after vascular procedures of the lower limb: accurate wound reclosure and 3 weeks of consistent and continuing drainage. Ann Vasc Surg 2012;26: 833-8.

10. Kobayashi $\mathrm{H}$, lida $\mathrm{T}$, Yamamoto $\mathrm{T}$, et al. Lymphaticovenous anastomoses for lymphedema complicated by severe lymphorrhea following resection of soft-tissue sarcomas of the adductor compartment: a report of two cases. JBJS Case Connect 2017;7:e80. 